\title{
Towards out-of-the-lab EEG in uncontrolled environments: feasibility study of dry EEG recordings during exercise bike riding
}

Link to publication record in Manchester Research Explorer

Citation for published version (APA):

Kohli, S., \& Casson, A. J. (2015). Towards out-of-the-lab EEG in uncontrolled environments: feasibility study of dry EEG recordings during exercise bike riding. In host publication IEEE.

\section{Published in:}

host publication

\section{Citing this paper}

Please note that where the full-text provided on Manchester Research Explorer is the Author Accepted Manuscript or Proof version this may differ from the final Published version. If citing, it is advised that you check and use the publisher's definitive version.

\section{General rights}

Copyright and moral rights for the publications made accessible in the Research Explorer are retained by the authors and/or other copyright owners and it is a condition of accessing publications that users recognise and abide by the legal requirements associated with these rights.

\section{Takedown policy}

If you believe that this document breaches copyright please refer to the University of Manchester's Takedown Procedures [http://man.ac.uk/04Y6Bo] or contact uml.scholarlycommunications@manchester.ac.uk providing relevant details, so we can investigate your claim.

\section{OPEN ACCESS}




\title{
Towards out-of-the-lab EEG in uncontrolled environments: feasibility study of dry EEG recordings during exercise bike riding
}

\author{
Siddharth Kohli and Alexander J. Casson, Member, IEEE
}

\begin{abstract}
Conventional EEG (electroencephalography) has relied on wet electrodes which require conductive gel to help the electrodes make contact with the scalp. In recent years many dry electrode EEG systems have become available that do not require this gel. As a result they are quicker and easier to set up, with the potential to record the the EEG in situations and environments where it has not previously been possible. This paper investigates the practicality of using dry EEG in new nonconventional recording situations. In particular it uses a dry EEG recording system to monitor the EEG while a subject is riding an exercise bike. The results show that good-quality EEG, free from high-amplitude motion artefacts, can be collected in this challenging motion rich environment. In the frequency domain a peak of activity is seen over the motor cortex (C4) at $23 \mathrm{~Hz}$ starting five minutes after the start of the exercise task, giving initial insights into the on-going operation of the brain during exercise.
\end{abstract}

\section{INTRODUCTION}

Since its discovery in 1929 [1] EEG (electroencephalography) has become an invaluable tool for the non-invasive monitoring of the human brain. Based upon placing small metal electrodes on the scalp it provides a high temporal resolution (but low spatial resolution) approach compared to other brain monitoring modalities such as fMRI (functional Magnetic Resonance Imaging) and PET (Positron Emission Tomography).

In addition, advances in electronics and miniaturization mean that EEG is ideal for portable brain monitoring [2]. A major limitation to this portability has always been the electrodes used to connect the EEG recorder to the scalp. Sintered $\mathrm{Ag} / \mathrm{AgCl}$ electrodes offer the best recording performance [3], [4], but are wet: they require a conductive gel to make a connection between the scalp and electrode; in addition to needing standard preparations such as parting the hair and cleaning the scalp.

As a result, conventional wet electrode EEG systems are very difficult to set up quickly and without the assistance of a trained technician. In recent years there has been substantial progress on dry EEG electrodes to mitigate these issues. These do not require conductive gel and can still collect high quality EEG signals; see [5] for a review of the electrode technologies avaiable. Such dry electrode EEG systems are now starting to be commercially available from companies such as g.tec, Mindo, Quasar and others [6].

To date, many studies on dry EEG electrodes have investigated the comparison of wet and dry electrodes by

The authors are with the School of Electrical and Electronic Engineering, The University of Manchester, UK. Email: siddharth.kohli@postgrad.manchester.ac.uk, alex.casson@manchester.ac.uk. reporting a correlation coefficient between EEG recorded at nearby locations with the two electrode types. Typical values reported are: $>0.93$ [7]; 0.89 [8]; 0.83 [9]; 0.81-0.98 [10]; 0.68-0.90 [11]; 0.39-0.85 [12]. However, in addition to being quicker and easier to set up, these novel electrodes also offer the potential to record the EEG in situations and environments where it has not previously been possible: a major aim of dry EEG systems is to open new avenues in where and when EEG monitoring can be performed. To complement the above wet/dry comparison studies it is thus now essential to demonstrate the applicability of dry electrode recording systems in new recording situations.

This paper investigates this potential of using new dry electrodes to record the EEG in non-conventional environments. Rather than performing a comparison of wet and dry electrodes, we make use of only dry electrodes and apply them in a situation where conventional EEG monitoring is not generally possible: to record brainwave signals from a subject while pedalling on an exercise bike. This is an active motion task with the potential for lots of motion interference.

Our results demonstrate that EEG signals can be collected in this situation, free from high-amplitude motion artefacts. Section II outlines the experimental protocol used, with the results presented in Section III. Further, the collected EEG data shows increased activity over the motor cortex (C4) in the mid- $\beta$ band ( $23 \mathrm{~Hz}$ region) after five minutes of bike riding activity. The implications of this are discussed in Section IV. Overall the results demonstrate the potential of emerging dry EEG systems for providing high time resolution brain monitoring in previously infeasible recording scenarios.

\section{METHODS}

Three dry EEG recordings were carried out using the Quasar DSI-mini EEG recorder illustrated in Fig. 1. This is a six channel, 16-bit, $300 \mathrm{~Hz}$ sampling rate EEG recorder which uses fingered electrodes in order to penetrate the hair and obtain a direct connection with the scalp without having to part the hair in advance. This is combined with an ultrahigh input impedance Common-Mode Follower approach [8] which allows EEG recordings to be performed even in the presence of a high impedance electrode connection (as no conductive gel is used to lower the impedance).

The DSI-mini EEG unit was set up and placed on the subject's head by a technician as in standard EEG recordings. (The potential for the subject to self-don the EEG unit was not investigated in this study.) EEG electrodes were placed in $10 / 20$ positions at $\mathrm{F} 7, \mathrm{~F} 8, \mathrm{C} 3, \mathrm{C} 4, \mathrm{~T} 3$, T4 with the ground 

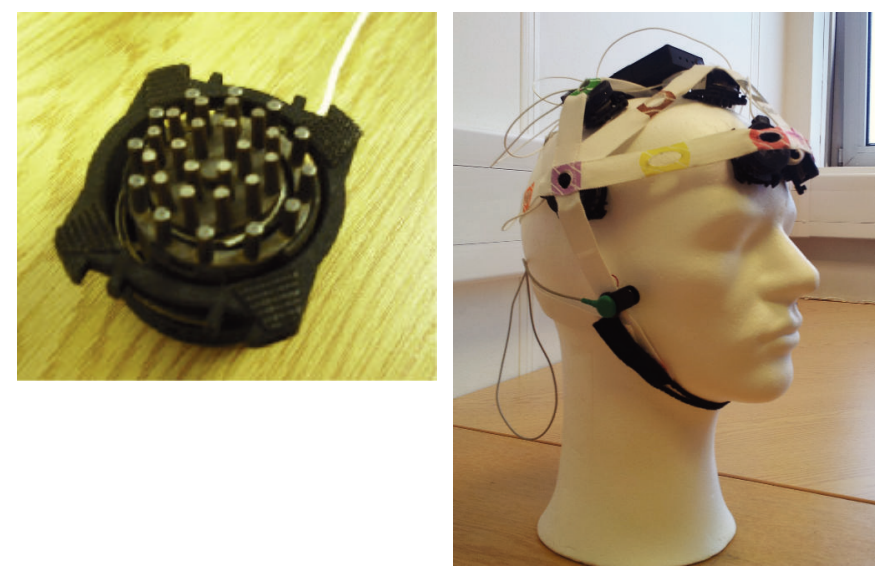

Fig. 1. The Quasar DSI-mini EEG recorder used in this experiment. This uses dry electrodes which are fingered to easily penetrate the hair and obtain a direct connection with the scalp.

connection at A2 and the common mode follower input at Fz. The subject was then asked to pedal on a standard exercise bike set at minimum resistance for a period of one hour. At all times the subject was in the standard sitting position for riding a bike and their upper body movement was not restrained in any way. Data was recorded at Imperial College London and the institution's Ethical Review Board approved all experimental procedures involving human subjects.

In Section III time domain analysis of the signals is performed by visual inspection for the presence of motion artefacts in the collected raw EEG signals. For further analysis the collected signals were bandlimited from $1-50 \mathrm{~Hz}$, with an additional $50 \mathrm{~Hz}$ notch filter to remove mains noise.
Frequency domain analysis is performed by segmenting the EEG into five minute non-overlapping epochs. Within each epoch the Welch transform is calculated using a $2 \mathrm{~s}$ window of data, with $50 \%$ overlap present between each window. A $2^{11}$ point FFT with a Hamming window is used as the basis for this transform. This approach allows the changes in EEG power between each five minute epoch to be investigated, whilst also giving multiple $2 \mathrm{~s}$ analysis windows that can be averaged together in order to improve the frequency domain signal-to-noise ratio and highlight the EEG frequencies that are consistently present during each five minute period.

\section{RESULTS}

Fig. 2 shows the unfiltered EEG collected during one of the pedalling trials. The EEG data is almost completely free of high-amplitude motion artefact in all of the channels for approximately the first half an hour. After this the data quality begins to degrade, most likely due to a build up of sweat on the scalp altering the electro-chemical equilibrium of the electrode connection, as is common in EEG recordings [13]. Air conditioning normally largely eliminates the presence of such artefacts in non-mobile subjects [14], but this is of course not possible in the active exercise task being carried out here.

From all of the EEG trials at least 20 minutes of good quality EEG was collected. Fig. 3 shows a shorter section of channel $\mathrm{C} 4$ from three EEG recordings, illustrating the typical EEG produced after bandlimiting filtering. The potential to record low amplitude free-running EEG signals is clearly demonstrated, despite the fact that the subject is pedalling

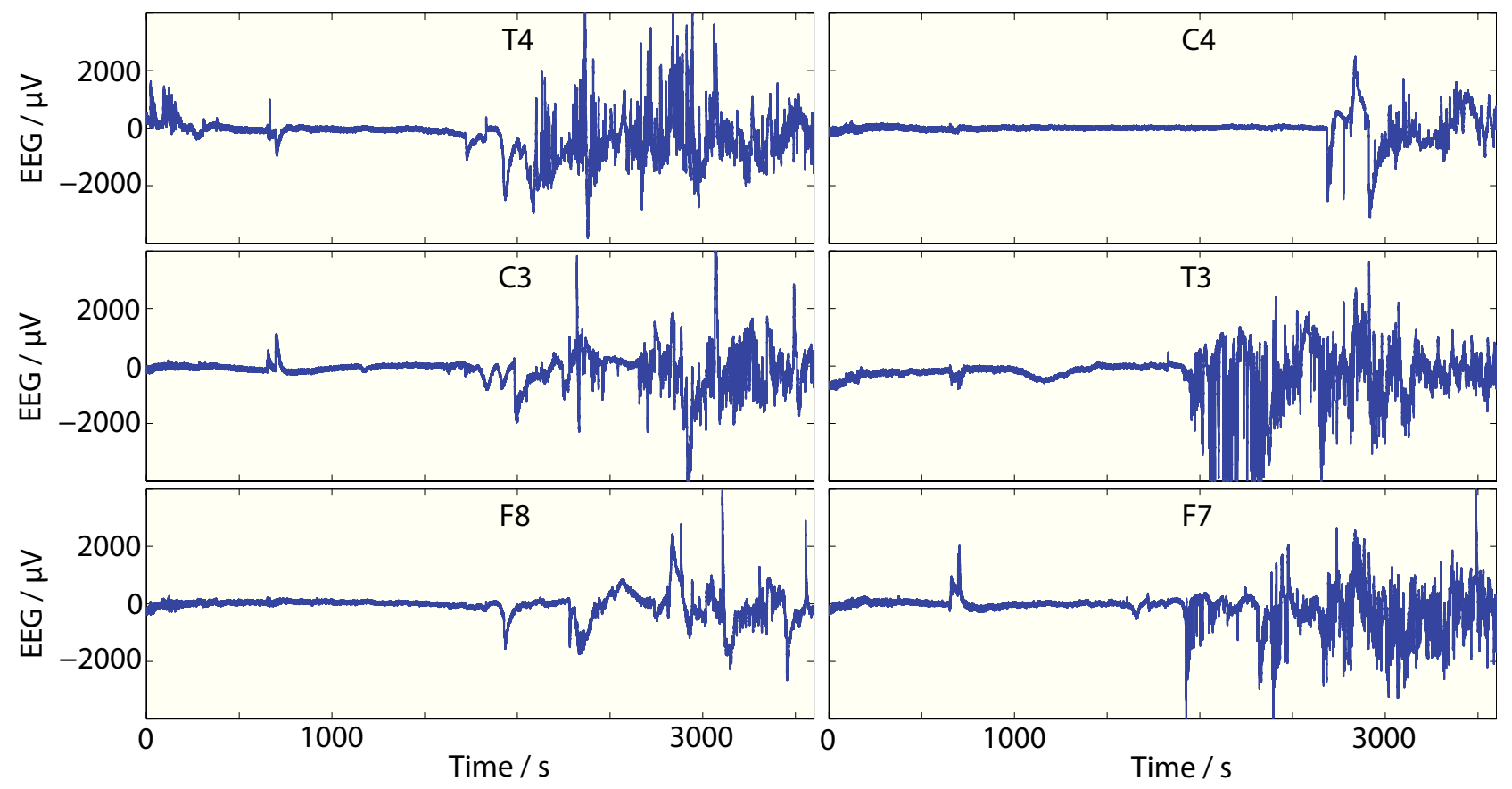

Fig. 2. The full EEG time series from one bike riding session; no filtering is applied. Good quality EEG data is collected in the first half an hour of recording, with very few high-amplitude motion artefacts present despite the ongoing bike riding activity. 


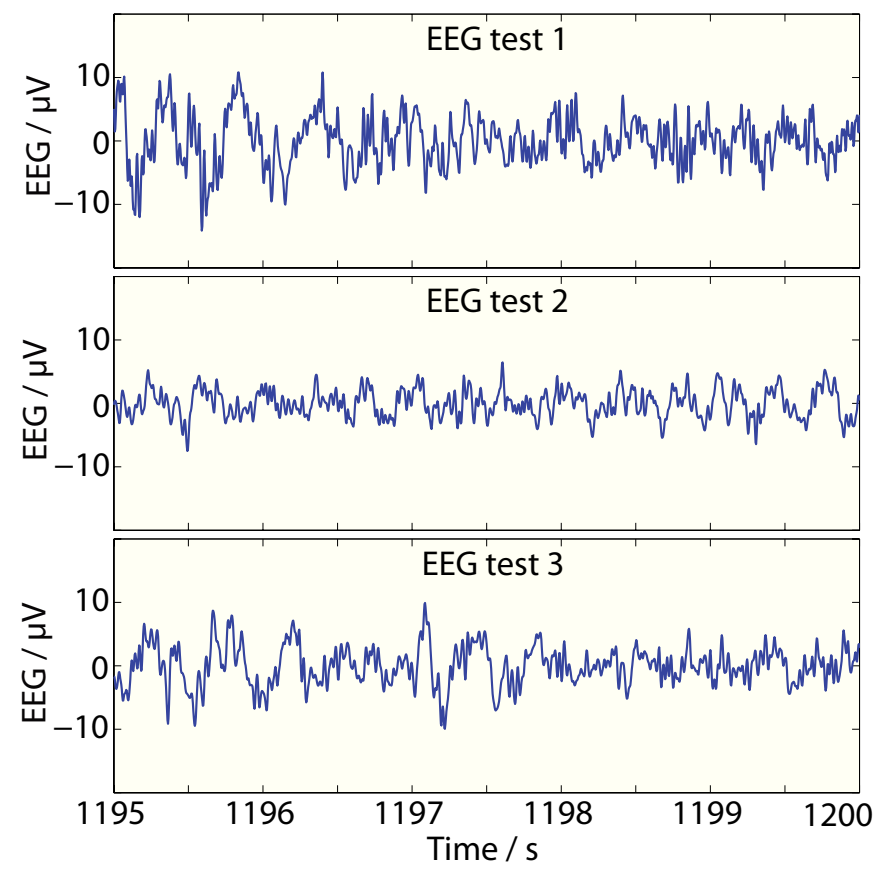

Fig. 3. Five seconds of EEG data from channel $\mathrm{C} 4$ in three seperate recordings. This illustrates the typical quality of EEG recorded after 20 minutes of bike riding activity.

on a bike during the collection of these signals and has been doing so for 20 minutes.

To demonstrate that these signals contain potentially useful neurophysiological information, Fig. 4 presents the EEG Power Spectral Densities (PSD) for the first 20 minutes of recording (while good quality EEG data is being collected in all of the cases). For illustration purposes only the results for the C4 and F8 electrodes are shown here. No sections of data have been rejected prior to calculating this PSD.

In channel $\mathrm{C} 4 \alpha / \mu$ band peaks are clearly observable in all of the cases. In addition, most clearly seen in test 3, after the first five minutes of recording there is also clear activation of the motor cortex $(\mathrm{C} 4)$ with a peak in frequency activity around $23 \mathrm{~Hz}$. This peak is maintained throughout the rest of the experiment. The $23 \mathrm{~Hz}$ peak is also present in test 2 from 5-20 minutes. In test case 1 small peaks in this mid- $\beta$ frequency range are present, but they are much less pronounced.

\section{DISCUSSION}

In Fig. 4, comparing channel $\mathrm{C} 4$ to $\mathrm{F} 8$, no peaks in the $\beta$ band are seen in F8 or in the (not illustrated) other nonmotor electrodes. This indicates that the mid- $\beta$ peaks do correspond to an activation of the motor cortex, as opposed to a more general activation of the brain, or the presence of broad motion artefacts. Voluntary muscle movement has long been associated with decreased $\beta$ power (increased $\beta$ desynchronisation) compared to a baseline resting case [15], [16]. No baseline resting case is illustrated in Fig. 4: instead Fig. 4 shows the evolution of the $\beta$ band after the start of exercise. It suggests a potential decrease in $\beta$ desynchronisation as the exercise task increases in duration, with more activation in the $\mathrm{C} 4$ region several minutes after the start of exercise.

At this point further insights into the origin of the $23 \mathrm{~Hz}$ peaks, and why they only emerge after the first 5 minutes of the exercise task, are not available. Potentially there is an extended electrode-settling time, with this transitory property of the electrodes masking underlying low level signals at the start of the experiment. (See [4] for a discussion of such transients in conventional EEG electrodes.) Alternatively a learning effect within the task, as the subject becomes familiar with the particular exercise bike used and settles into the process of riding it, cannot be ruled out. No period of acclimatisation was incorporated into the experimental protocol.

Nevertheless, the high time resolution neurophysiological dynamics of cycling - and moreover the temporal evolution of the EEG and brain state-have not been well studied previously. They can only be done so now due to the emergence of high quality dry electrode EEG approaches. As a result there are few comparison results available for validating the EEG trends identified here. [17] used PET scanning to monitor subjects while cycling and found a significant activation of the primary motor cortex during rhythmic cycling motion, consistent with the results here.

To our knowledge, the only EEG based study is [16] which used a reclining stationary bicycle allowing them to restrain the user to minimise motion artefact and then use sponge based electrodes to record the EEG. Analysis in [16] focused on the $\mathrm{C} 1, \mathrm{Cz}, \mathrm{C} 2$ region and showed a pedalling related potential at twice the pedalling frequency when the EEG from each cycle of the feet was averaged together. They also compared $\beta$ band powers between rest and active movement and found no change in $\beta$ desynchronisation. A significant difference in desynchronisation was observed between trials where the subject pedalled and trails where the pedals were moved for the subject, with just their feet resting on them. However, only the first $500 \mathrm{~s}$ (8 minutes) of EEG data was used in this study, insufficient to investigate the temporal evolution of EEG powers as done here.

The current study has thus demonstrated the potential utility of using dry EEG electrodes for high temporal resolution brain monitoring in non-conventional situations. This has allowed the collection of preliminary results that suggest an increase in mid- $\beta$ activity during prolonged exercise.

\section{CONCLUSIONS}

Dry EEG electrodes are now opening new avenues in where and when EEG monitoring can be performed. This study has used them to allow monitoring of the EEG during a bike riding task and the results demonstrate that good quality EEG signals can be collected which are not dominated by high-amplitude motion artefacts. Frequency domain analysis of these signals shows clear peaks in the $\alpha / \mu$ band together with a relative increase in mid- $\beta$ activity occurring five minutes after the start of the cycling task. Inevitably further 


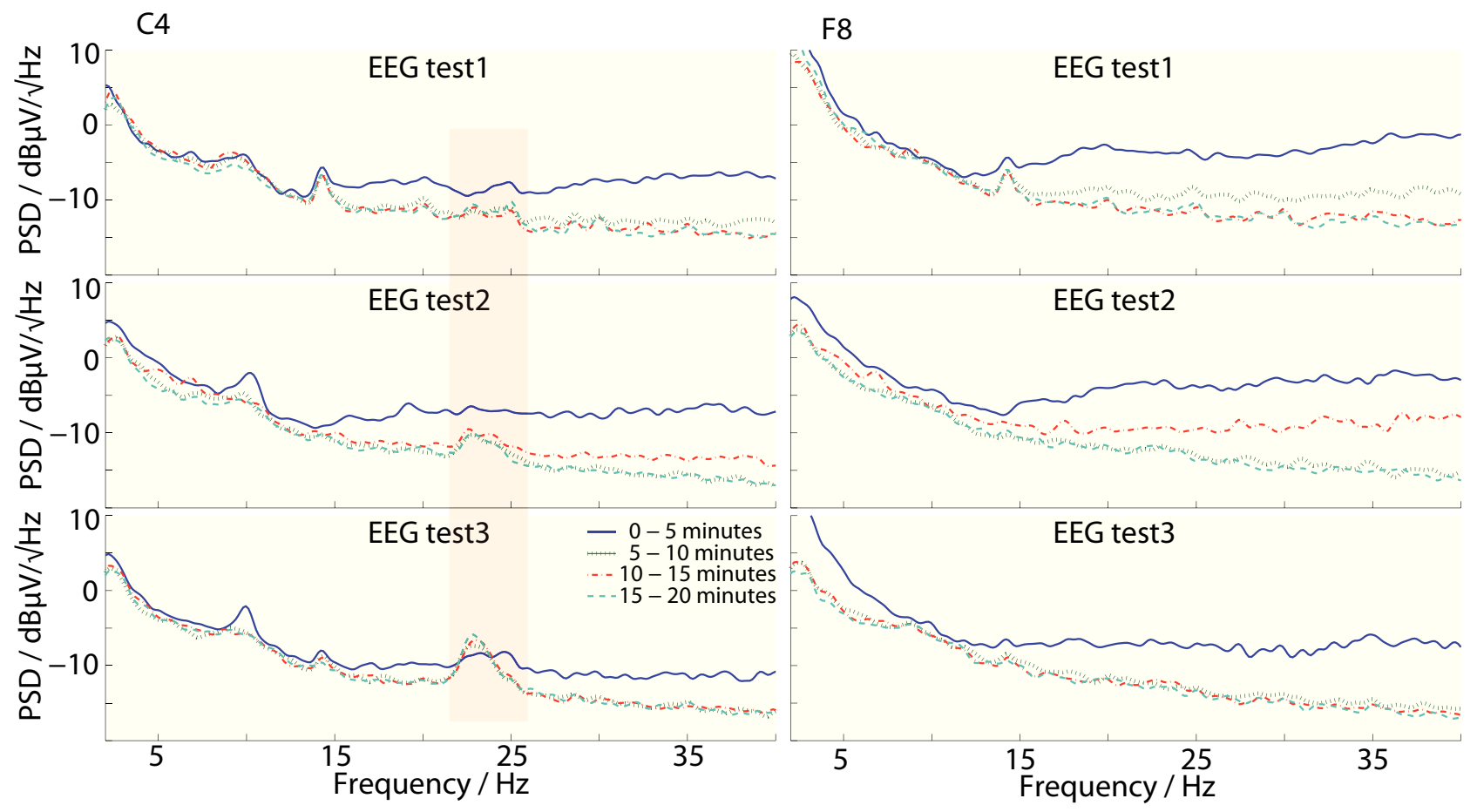

Fig. 4. EEG Power Spectral Densities (PSD) found during the first 20 minutes of the bike riding task. This is calculated in non-overlapping five minute epochs to show the PSD evolution over time. Left: EEG channel C4; Right: EEG channel F8. Highlighted is the region around $23 \mathrm{~Hz}$ in channel C4 which shows a clear elevation in tests 2 and 3, with smaller peaks present in test 1. No such activations are present in the F8 channel.

experimentation is necessary to validate these neurophysiological changes, but this paper has demonstrated that it is now possible to use dry EEG recordings to enable EEG monitoring in non-conventional situations. This opens new avenues for brain research using portable EEG monitors, and to moves electrode research beyond only comparing the performance of wet and dry electrodes. As a result we are now gaining initial insights into the on-going operation of the brain during exercise.

\section{ACKNOWLEDGEMENTS}

The authors would like to acknowledge and thank Quasar USA (http://quasarusa.com/) for the loan of the DSI-mini EEG recorder used in this study.

\section{REFERENCES}

[1] H. Berger, "Uber das eletrenkephalogram des menschen," Arch. Psychiatr. Nervenkr, vol. 87, no. 1, pp. 527-570, 1929.

[2] A. J. Casson, D. C. Yates, S. J. Smith, J. S. Duncan, and E. RodriguezVillegas, "Wearable electroencephalography," IEEE Eng. Med. Biol. Mag., vol. 29, no. 3, pp. 44-56, 2010.

[3] E. Huigen, A. Peper, and C. A. Grimbergen, "Investigation into the origin of the noise of surface electrodes," Med. Biol. Eng. Comput., vol. 40, no. 3, pp. 332-338, 2002.

[4] E. Camacho-Galeano, C. Galup-Montoro, and M. Schneider, "Evaluation of commercially available electrodes and gels for recording of slow EEG potentials," Clin. Neurophysiol., vol. 116, no. 4, pp. 799806, 2005.

[5] Y. Chi, T.-P. Jung, and G. Cauwenberghs, "Dry-contact and noncontact biopotential electrodes: Methodological review," IEEE Rev. Biomed. Eng., vol. 3, no. 1, pp. 106-119, 2010.

[6] A. J. Casson, "Towards noise-enhanced augmented cognition," in $\mathrm{HCI}$ international, Las Vegas, July 2013.
[7] J. Xu, R. F. Yazicioglu, B. Grundlehner, P. Harpe, K. A. A. Makinwa, and C. Van Hoof, "A $160 \mu \mathrm{W}$ 8-channel active electrode system for EEG monitoring," IEEE Trans. Biomed. Circuits Syst., vol. 5, no. 6, pp. 555-567, 2011.

[8] R. Matthews, N. J. McDonald, P. Hervieux, P. J. Turner, and M. A. Steindorf, "A wearable physiological sensor suite for unobtrusive monitoring of physiological and cognitive state," in IEEE EMBC, Lyon, August 2007.

[9] G. Gargiulo, P. Bifulco, R. A. Calvo, M. Cesarelli, C. Jin, and A. van Schaik, "A mobile EEG system with dry electrodes," in IEEE BioCAS, Baltimore, November 2008.

[10] IMEC. (2012) Holst centre and panasonic present wireless lowpower active-electrode EEG headset. [Online]. Available: http: //www.imec.be/

[11] S. Patki, B. Grundlehner, A. Verwegen, S. Mitra, J. Xu, A. Matsumoto, R. F. Yazicioglu, and J. Penders, "Wireless EEG system with real time impedance monitoring and active electrodes," in IEEE BioCAS, Hsinchu, November 2012.

[12] J. R. Estepp, J. C. Christensen, J. W. Monnin, I. M. Davis, and G. F Wilson, "Validation of a dry electrode system for EEG," in Proc. HFES, San Antonio, October 2009.

[13] W. O. Tatum, B. A. Dworetzky, and D. L. Schomer, "Artifact and recording concepts in EEG," J. Clin. Neurophysiol., vol. 28, no. 3, pp. 252-263, 2011

[14] J. W. Britton, "Electroencephalographic artifacts and benign variants," in Aminoff's Electrodiagnosis in Clinical Neurology, 6th ed., M. J. Aminoff, Ed. Amsterdam: Elsevier Saunders, 2012, pp. 129-142.

[15] G. E. Chatrian, M. C. Petersen, and A. Lazarte, J, "The blocking of the rolandic wicket rhythm and some central changes related to movement," Electroencephalogr. and Clin. Neurophysiol., vol. 11, no. 3, pp. 497-510, 1959.

[16] S. Jain, K. Gourab, S. Schindler-Ivens, and B. D. Schmit, "EEG during pedaling: Evidence for cortical control of locomotor tasks," Clin. Neurophysiol., vol. 124, no. 2, pp. 379-390, 2013.

[17] L. Christensen, P. Johannsen, T. Sinkjaer, N. Petersen, H. Pyndt, and J. Nielsen, "Cerebral activation during bicycle movements in man," Exp. Brain Res., vol. 135, no. 1, pp. 66-72, 2000. 